\title{
Psoriatic arthritis: A permanent new challenge for dermatologists (Review)
}

\author{
ALINA DINU $^{1 *}$, STEFANA BUCUR $^{1 *}$, RODICA OLTEANU $^{1 *}$, TRAIAN CONSTANTIN $^{2,3 *}$, \\ ANCA RADUCAN $^{4 *}$, MARA BAETU $^{5^{*}}$ and MARIA-MAGDALENA CONSTANTIN ${ }^{1,3^{*}}$ \\ ${ }^{1}$ Second Department of Dermatology, Colentina Clinical Hospital, 20125 Bucharest; ${ }^{2}$ Department of Urology, \\ 'Th. Burghele' Hospital, 50652 Bucharest; ${ }^{3}$ General Medicine, University of Medicine and Pharmacy 'Carol Davila', \\ 050474 Bucharest; ${ }^{4}$ Dr. Anca Răducan Anti-Aging Dermatology Clinic, 900705 Constanta; \\ 5،C.I. Parhon' National Institute of Endocrinology, 011863 Bucharest, Romania
}

Received September 27, 2019; Accepted October 30, 2019

DOI: $10.3892 /$ etm.2019.8322

\begin{abstract}
Considering that most of the patients ( $>2 / 3)$ are diagnosed with psoriasis in the cutaneous form long before the joint damage occurs and, in these conditions, a significant proportion of them is found in the dermatologist's initial records, a question must be asked: when is it necessary to send these patients to a rheumatology consultation? The recognition of psoriatic arthritis in patients with vulgar psoriasis and the dermatologist's ability to differentiate it from other arthritis, offers the opportunity to improve patient prognosis by prompt intervention and close collaboration with the rheumatologist. Diagnosis of early psoriatic arthropathy should be considered when a patient with psoriasis or family history of psoriasis has peripheral inflammatory arthritis (oligoarthritis or distal interphalangeal joints damage), enthesitis, dactylitis, spinal pain of inflammatory type. Given that patients with psoriasis are included in the dermatologists' medical records, it is very important to recognize psoriatic arthritis in patients with cutaneous psoriasis, to differentiate it from other possible arthritis, thus having the possibility to improve patient prognosis by prompt intervention and through collaboration with the rheumatologist.
\end{abstract}

\section{Contents}

1. Introduction

2. Materials and methods

3. Spondylarthritis classification

4. General perspectives on psoriatic arthritis

Correspondence to: Dr Stefana Bucur, Second Department of Dermatology, Colentina Clinical Hospital, 19-21 Stefan cel Mare Street, 20125 Bucharest, Romania

E-mail: stefanabucur11@gmail.com

\section{${ }^{*}$ Contributed equally}

Key words: psoriasis, psoriatic arthritis, joints pain, enthesitis, dactylitis
5. Classification and diagnosis of psoriatic arthropathy

6. Psoriasis associations with other diseases

7. Treatment

8. Conclusions

\section{Introduction}

Considering that most of the patients $(>2 / 3)$ are diagnosed with psoriasis in the cutaneous form long before the joint damage occurs and, in these conditions, a significant proportion of them is found in the dermatologist's initial records, a question must be asked: when is it necessary to send these patients to a rheumatology consultation?

Moreover, in the absence of a set of specific criteria for the diagnosis of psoriatic arthritis, the most commonly used method for recognizing and monitoring this condition remains the clinical aspect.

Thus, the recognition of psoriatic arthritis in patients with vulgar psoriasis and the dermatologist's ability to differentiate it from other arthritis, offers the opportunity to improve patient prognosis by prompt intervention and close collaboration with the rheumatologist (1).

\section{Materials and methods}

As far as psoriatic arthritis is concerned, it can be defined as a seronegative inflammatory arthritis (absent rheumatoid factor) and characterized by the presence of: a) enthesitis, which is the inflammation of the entheses, the mark of psoriatic arthritis, b) dactylitis, which is the diffuse swelling of a finger and can be acute (with painful inflammatory changes) or chronic (the 'sausage finger') and it represents the cardinal feature of psoriatic arthritis, c) axial damage and d) the presence of HLA-B27 antigen (the prevalence of HLA-B27 in psoriatic arthritis patients ranged from 20 to $35 \%$ in some studies) (2).

\section{Spondylarthritis classification}

Classification of spondylarthritis is divided in two major subtypes: a) predominantly peripheral spondylarthritis, which 
includes: arthritis associated with previous acute uveitis, psoriatic arthritis, reactive arthritis (Reiter), juvenile idiopathic arthritis, arthritis associated with intestinal inflammatory diseases and undifferentiated spondylarthritis and b) predominantly axial spondylarthritis, with ankylosing spondylitis (3). Under these conditions, psoriatic arthropathy is considered a peripheral spondylarthritis associated with HLA-B27 antigen.

Classification criteria for predominantly peripheral spondylarthritis apply to subjects who at the time of examination have at least one of these affections: arthritis, enthesitis or dactylitis. If the patient meets one of these three features, then he or she must at least have one feature from Group A or at least two features from Group B. ASAS criteria for classification of peripheral spondylarthritis can be seen in Fig. 1 (4).

\section{General perspectives on psoriatic arthritis}

From an epidemiological point of view, psoriatic arthritis occurs in $6-24 \%$ of patients with vulgar psoriasis, predominantly similar in males and females, with onset at $35-45$ years $(5,6)$.

It is of interest to mention that $70 \%$ of cases of skin psoriasis precede joint damage, there is a simultaneous debut in $10-15 \%$ of cases and in $15 \%$ of cases, arthritis precedes cutaneous damage. Regarding children, arthritis precedes the occurrence of skin psoriasis in $>50 \%$ of cases, with female sex being more frequently affected (7).

Nail lesions are very common and help distinguish between patients who have psoriatic arthritis and those who have rheumatoid arthritis and between patients with psoriasis who have arthritis and those who do not have arthritis. Nail lesions occur in about $40-45 \%$ of patients with psoriasis uncomplicated by arthritis and $\sim 87 \%$ of patients with psoriatic arthritis (8). Although the etiology of psoriatic arthritis has not been fully elucidated, we would like to mention the role of beta blockers which are a widely used class of medication. Their use in dermatology has garnered growing interest with the discovery of their therapeutic effects in the treatment of haemangiomas, their potential positive effects in wound healing, Kaposi sarcoma, melanoma and pyogenic granuloma, and, more recently, pemphigus. However, like any other drug category, they carry risks of side effects, some of which are dermatologic. These include triggering and exacerbation of psoriasis, psoriatic and rheumatoid arthritis, anaphylaxis, contact dermatitis, occupational contact dermatitis, Raynaud's disease, alopecia, lichen planus-like drug eruption, hyperhidrosis and vitiligo $(9,10)$.

\section{Classification and diagnosis of psoriatic arthropathy}

Clinical and laboratory criteria for the classification and diagnosis of psoriatic arthropathy were enunciated by Moll and Wright over 30 years ago and are still the simplest and most commonly used in practice. These are represented by the inflammatory arthritis, the presence of psoriasis and the absence of serum rheumatoid factor.

Using these criteria, Moll and Wright (11) described 5 clinical subtypes: a) Asymmetric oligoarthritis, which is characterized by damage to the proximal and distal interphalangeal joints of the hands and feet, with the appearance of the 'sausage finger' or asymmetric dactylitis. Metacarpophalangeal and metatarsophalangeal joints may be affected, but also the damage to the knee, tibiotarsal and radiocarpal joints may occur; b) Psoriatic arthritis is the second subtype presented and the most frequent onset manifestation, having multiple similarities with rheumatoid arthritis, for example, symmetry and the damage to small joints. There are several elements that differentiate them, therefore, psoriatic arthritis is characterized by radiocubitocarpal and distal interphalangeal injury, evolutionary conversion and the subsequent damage is limited to a few joints; c) Distal interphalangeal arthritis, which is associated with nail psoriasis lesions; d) Arthritis mutilans, which is a rare form as a manifestation of the onset of the disease. It is an extensive osteolytic form of phalanges, usually the distal ones; e) Spondyloarthropathy, which associates sacroiliitis and spinal cord injury with oligoarticular peripheral arthritis, not forgetting that sacroiliitis is unilateral and the evolution in this case is unfavorable, especially in males (12).

However, the Moll and Wright criteria are not sufficient for an early and accurate diagnosis of psoriatic arthropathy. Over the years, a set of criteria as specific as possible for the diagnosis and classification of psoriatic arthropathy has been attempted, these trials have culminated with the publication in 2006 of CASPAR Criteria (Criteria for Classification of Psoriatic Arthropathy (13). Thus, in order to meet CASPAR criteria, a patient should present inflammatory arthritis (at the peripheral joints, spinal damage or enthesis damage) and at least three of the following: a) Psoriasis at the time of examination; personal history of psoriasis (if there are no current injuries); family history of psoriasis in first or second degree relatives (if there are no current lesions or personal history of psoriasis); b) Nail changes (pitting, onycholysis, hyperkeratosis); c) The absence of rheumatoid factor; d) Dactylitis - swelling of a finger; e) Juxta-articular bone neoformation (except osteophytes).

Thus, an early diagnosis of psoriatic arthropathy is difficult to achieve also due to very different clinical manifestations. Also, articular inflammation may be clinically silent (it is sometimes detected when articular destruction is clinically or imaging evident) and, furthermore, no clinical, immunohistochemical or genetic specific marker has been identified so far to indicate patients with psoriasis that will develop inflammatory arthritis (Table I).

\section{Psoriasis associations with other diseases}

Psoriasis can be associated with any other spondyloarthropathy: a) Reactive arthritis. It occurs predominantly between the age of 20 and 40 years, which coincides with the onset of psoriatic arthritis, but the relatively early onset of arthritis and enthesitis is differentiated a few days after a trigger infection (Campylobacter, Chlamydia T, Yersinia, Shigella, Salmonella) (14). It is worth mentioning the appearance of Reiter syndrome, which is characterized by the appearance of arthritis, conjunctivitis and urethritis. Cutaneous mucosal manifestations of keratoderma blennorrhagica (small and hard nodules occurring frequently on soles, but also on the hands) may also occur.

b) Osteoarthritis: It affects older people more frequently than psoriatic arthritis. Heberden and Bouchard nodules can 
Table I. Differential diagnosis of psoriatic arthritis.

\begin{tabular}{|c|c|c|c|c|c|c|}
\hline $\begin{array}{l}\text { Clinical } \\
\text { examination }\end{array}$ & $\begin{array}{l}\text { Psoriatic } \\
\text { arthritis }\end{array}$ & Osteoarthritis & Gout & $\begin{array}{l}\text { Rheumatoid } \\
\text { arthritis }\end{array}$ & $\begin{array}{l}\text { Ankylosing } \\
\text { spondylitis }\end{array}$ & $\begin{array}{l}\text { Reactive } \\
\text { arthritis }\end{array}$ \\
\hline Injured joints & $\begin{array}{l}\text { Oligoarthritis } \\
\text { Polyarthritis } \\
\text { Axial }\end{array}$ & $\begin{array}{l}\text { Monoarthritis } \\
\text { Oligoarthritis }\end{array}$ & $\begin{array}{l}\text { Monoarthritis } \\
\text { Oligoarthritis } \\
\text { Polyarthritis }\end{array}$ & Polyarthritis & $\begin{array}{l}\text { Axial } \\
\text { Large peripheral } \\
\text { joints }\end{array}$ & $\begin{array}{l}\text { Oligoarthritis } \\
\text { Polyarthritis } \\
\text { Axial }\end{array}$ \\
\hline Distribution & $\begin{array}{l}\text { Peripheric } \\
\text { Axial }\end{array}$ & $\begin{array}{l}\text { Peripheric } \\
\text { Axial }\end{array}$ & Peripheric & Peripheric & Axial>Peripheric & $\begin{array}{l}\text { Peripheric } \\
\text { Axial }\end{array}$ \\
\hline Symmetry & Asymmetric & Asymmetric & Asymmetric & Symmetric & Symmetric & Asymmetric \\
\hline $\begin{array}{l}\text { Tumefaction/ } \\
\text { erythema }\end{array}$ & Present & Absent & Present & Present & Absent & Present \\
\hline Enthesitis & Present & Absent & Absent & Absent & Present & Present \\
\hline Dactylitis & $48 \%$ & Absent & Absent & Absent & Absent & Sometimes \\
\hline Synovitis & Present & Absent & Absent & Present & Present & Present \\
\hline
\end{tabular}

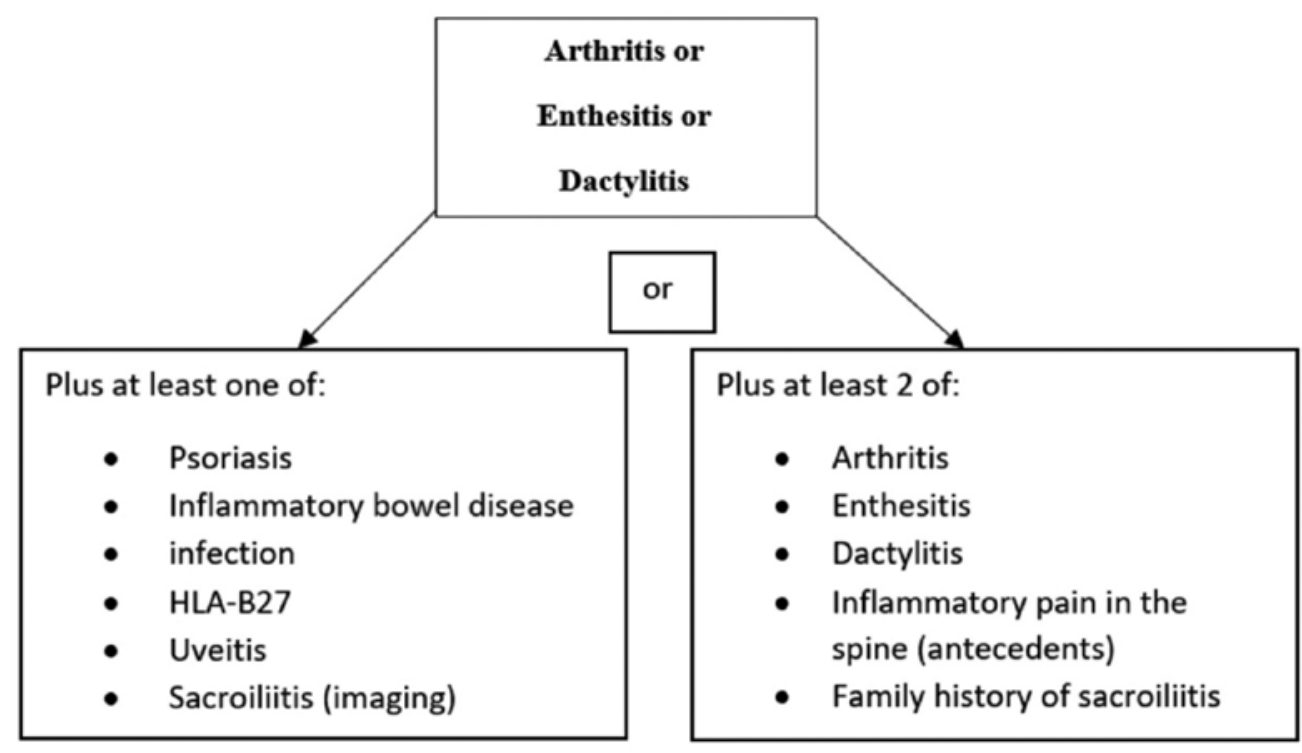

Figure 1. ASAS criteria for classification of peripheral spondylarthritis.

differentiate distal interphalangeal damage and the damage of proximal interphalangeal joints in psoriatic arthritis. Moreover, enthesitis and extraarticular manifestations do not occur in osteoarthritis (1).

c) Gout: As in the case of osteoarthritis, older people are also predominantly affected. Unlike psoriatic arthritis, gout attacks have an acute onset with severe pain, followed by a mandatory remission period. Monoarticular damage can be easily differentiated from psoriatic arthropathy, but diagnostic difficulties appear in polyarticular gout because the appearance may sometimes mimic clinical manifestations in psoriatic arthropathy. Note that although the swelling of a finger in gout may present similarities with dactylitis, enthesitis is not a characteristic of gout. Also, gout is frequently associated with elevated serum uric acid levels and it is radiographically differentiated from psoriatic arthropathy through C-shaped erosions and the absence of periostitis (14). d) Rheumatoid arthritis: It is defined as an inflammatory arthritis that has numerous similarities with psoriatic arthropathy such as pain, swelling, morning stiffness, joint damage and extraarticular manifestations, but enthesitis is not a feature of rheumatoid arthritis. Moreover, 'swan neck', zig-zag deformities, contribute to its differentiation from psoriatic arthropathy. Serological markers, integrated into the clinical context, bring an additionally contribution to differentiate these two disorders (14). Rheumatoid factor is present in $>2 / 3$ of patients with rheumatoid arthritis, having an important role in its diagnosis. The presence of rheumatoid factor in $5 \%$ of healthy adults should be noted though. However, citrulline antiprotein antibodies significantly increase the sensitivity and the specificity of rheumatoid arthritis diagnosis.

Rheumatoid arthritis can generally be diagnosed based on clinical, serological, and radiological criteria alone 
and, for clinical routine purposes, does not necessitate a biopsy $(15,16)$. Outside the research setting, a synovial biopsy can be justified in cases of unclear arthritis (17).

e) Ankylosing spondylitis: In this case, we encounter an earlier onset compared to psoriatic arthropathy, being characterized by a predilection for axial manifestations with bilateral sacroiliitis and spinal cord injury. Unlike psoriatic arthropathy, it is an irreversible disease that is associated with intense pain, stiffness and limitation of axial movements. Furthermore, it is characterized more frequently by extra-articular manifestations such as uveitis, urethritis and intestinal inflammatory disease (14).

\section{Treatment}

Among the drugs used according to the protocol in psoriatic arthropathy are the ones of first choice: non-steroidal anti-inflammatory drugs (NSAIDs), followed by Disease-modifying anti-rheumatic drugs (DMARDs) and then, biologic or synthetic drugs with target activity. Most recommendations promote an 'escalation therapy' strategy.

Regarding NSAIDS and DMARDS, their long-term use cannot be sustained due to inadequate pain relief, immune disturbances and serious gastrointestinal and cardiovascular adverse events. Therefore, other therapies with anti-inflammatory properties and minimum side effects are needed for the treatment of arthritis. In this category curcumin can also be included, it is a chemical produced by Curcuma longa plants. Curcumin has anti-inflammatory and antioxidant properties and modulates pro-inflammatory cytokines, adhesion molecules and CRP, thus eliciting a beneficial anti-inflammatory effect in arthritis, by reducing pain and CRP level, and increasing the walking distance, at a dosage of $200 \mathrm{mg}$ daily for 3 months (18).

Regarding biologic therapies, in recent years, there has been an increasing interest in developing new biologic treatments for psoriasis and psoriatic arthritis, therefore, there are many therapies available in Romania, among which: anti-TNF- $\alpha$ inhibitors, anti-IL-12/23 inhibitors and antiIL-17 inhibitors. Among anti-TNF- $\alpha$ inhibitors, the general safety profile of etanercept when it comes to the risk of infection and hepatotoxicity in patients with psoriasis, psoriatic arthritis and rheumatoid arthritis is higher than that of the other anti-TNF- $\alpha$ agents and, unlike the other anti-TNF agents, neutralizes the biological activity of both TNF- $\alpha$ and lymphotoxin- $\alpha(19,20)$.

An interesting study involving psoriasis treatment with ustekinumab, an interleukin-12/23 inhibitor, was conducted on 15 patients, with a mean age of 50.6 years and a male/female ratio of 2:1. Five patients had previous therapy as follows: 2 had adalimumab, 1 had etanercept, 1 had infliximab in a single therapy and one had infliximab and adalimumab. One third (5/15) of patients had arthritis. Patients with arthritis were significantly older than those without arthritis and no differences in age were seen by gender or previous treatment. Psoriasis area and severity index (PASI) and dermatological life quality index (DLQI) scores were measured at 1, 3, 6, 9 and 12 months, PASI 75 being the primary endpoint. A significant improvement in both PASI and DLQI scores was seen in the first 16 weeks, with a plateau until week 52. All patients achieved PASI 75 and $93.3 \%$ PASI 90 by month 12 (21). In conclusion, ustekinumab has demonstrated real efficacy in treatment of psoriasis and psoriatic arthritis.

Regarding biosimilar therapies, these are new drugs, highly similar copies of biological medicines, equally effective and safe. They have demonstrated real efficacy in treatment of psoriasis and psoriatic arthritis and represent a new trend in the treatment of many immune-mediated inflammatory diseases (22). Therefore, in the ever-expanding market of biosimilars, it is important for us as clinicians to be confident about the requirements for the approval of biosimilars. This reassurance comes from the evidence that has been generated before marketing authorization, and in particular from RCTs as an important part of it. Unfortunately, at present there is rather limited evidence provided by the clinical trials, especially on psoriasis and psoriatic arthritis, which would help a dermatologist feel more comfortable about prescribing biosimilars (20).

Research on the efficacy of complementary and alternative medicines (CAM) for psoriasis is increasing (23), but patients may misunderstand the benefits of these therapies. The results demonstrate that patients with psoriasis frequently use CAM because of traditional medication failures or side effects. Few turned to CAM because of limited care access, indicating that patients use CAM despite the availability of traditional treatments $(24,25)$.

For the patients with psoriatic arthritis, the heterogeneity and complexity of psoriatic arthritis pathogenesis requests complex methodological approaches. Going deeper in the pathogenesis of this disease, the associated genetic predisposition can lead to new immune biomarkers whether from the proteomic or genomic area and identify new therapeutic approaches (19).

\section{Conclusions}

Diagnosis of early psoriatic arthropathy should be considered when a patient with psoriasis or family history of psoriasis has peripheral inflammatory arthritis (oligoarthritis or distal interphalangeal joints damage), enthesitis, dactylitis, spinal pain of inflammatory type. Given that patients with psoriasis are included in the dermatologists' medical records (26), it is very important to recognize psoriatic arthritis in patients with cutaneous psoriasis, to differentiate it from other possible arthritis, thus having the possibility to improve patient prognosis by prompt intervention and through collaboration with the rheumatologist.

\section{Acknowledgements}

Not applicable.

\section{Funding}

Not applicable.

\section{Availability of data and materials}

All data generated or analyzed during this study are included in this published article. 


\section{Authors' contributions}

MMC contributed in all the stages of the article, she designed the article and revised the manuscript for important intellectual content. Four reviewers: SB, AR, TC, MB acquired the data by screening the papers identified on Pubmed. AD revised the work critically. RO, SB, AR drafted the work. MB and RO had substantial contributions to the analysis and interpretation of data for the work. AD contributed to the conception of the work and revised the language. All authors agreed to be accountable for all aspects of the work in ensuring that questions related to the accuracy or integrity of any part of the work are appropriately investigated and resolved. All authors read and approved the final manuscript.

\section{Ethics approval and consent to participate}

Not applicable.

\section{Patient consent for publication}

Not applicable.

\section{Competing interests:}

The authors declare that they have no competing interests and they have no financial relationships to disclose.

\section{References}

1. Garg A and Gladman D: Recognizing psoriatic arthritis in the dermatology clinic. J Am Acad Dermatol 63: 733-748, quiz 749-750, 2010.

2. Queiro R, Morante I, Cabezas I and Acasuso B: HLA-B27 and psoriatic disease: A modern view of an old relationship. Rheumatology (Oxford) 55: 221-229, 2016.

3. Gladman DD: Psoriatic arthritis. Dermatol Ther 17: 350-363, 2004.

4. Rudwaleit M, van der Heijde D, Landewé R, Akkoc N, Brandt J, Chou CT, Dougados M, Huang F, Gu J, Kirazli Y, et al: The Assessment of SpondyloArthritis International Society classification criteria for peripheral spondyloarthritis and for spondyloarthritis in general. Ann Rheum Dis 70: 25-31, 2011.

5. Helmick CG, Felson DT, Lawrence RC, Gabriel S, Hirsch R, Kwoh CK, Liang MH, Kremers HM, Mayes MD, Merkel PA, et al, National Arthritis Data Workgroup: Estimates of the prevalence of arthritis and other rheumatic conditions in the United States Part I. Arthritis Rheum 58: 15-25, 2008.

6. Leung YY, Tam LS, Kun EW and Li EK: Psoriatic arthritis as a distinct disease entity. J Postgrad Med 53: 63-71, 2007.

7. Gladman DD, Antoni C, Mease P, Clegg DO and Nash P Psoriatic arthritis: Epidemiology, clinical features, course, and outcome. Ann Rheum Dis 64 (Suppl 2): ii14-ii17, 2005.
8. Gladman DD, Anhorn KA, Schachter RK and Mervart H: HLA antigens in psoriatic arthritis. J Rheumatol 13: 586-592, 1986

9. Tatu AL, Elisei AM, Chioncel V, Miulescu M and Nwabudike LC: Immunologic adverse reactions of $\beta$-blockers and the skin. Exp Ther Med 18: 955-959, 2019.

10. Tatu AL and Nwabudike LC: Metoprolol-associated onset of psoriatic arthropathy. Am J Ther 24: e370-e371, 2017.

11. Moll JM and Wright V: Psoriatic arthritis. Semin Arthritis Rheum 3: 55-78, 1973.

12. Gladman DD: Axial disease in psoriatic arthritis. Curr Rheumatol Rep 9: 455-460, 2007.

13. Taylor W, Gladman D, Helliwell P, Marchesoni A, Mease P and Mielants H; CASPAR Study Group: Classification criteria for psoriatic arthritis: Development of new criteria from a large international study. Arthritis Rheum 54: 2665-2673, 2006.

14. Gladman D, Ritchlin C and Sieper J: Clinical manifestations and diagnosis of psoriatic arthritis. UpToDate, 2018. https://www. uptodate.com/contents/clinical-manifestations-and-diagnosis-of -psoriatic-arthritis.

15. Gerlag D and Tak PP: Synovial biopsy. Best Pract Res Clin Rheumatol 19: 387-400, 2005.

16. Gerlag DM and Tak PP: How useful are synovial biopsies for the diagnosis of rheumatic diseases? Nat Clin Pract Rheumatol 3: 248-249, 2007.

17. Saaibi DL and Schumacher HRJ Jr: Percutaneous needle biopsy and synovial histology. Baillieres Clin Rheumatol 10: 535-554, 1996.

18. Constantin MM, Nita IE, Olteanu R, Constantin T, Bucur S, Matei $\mathrm{C}$ and Raducan A: Significance and impact of dietary factors on systemic lupus erythematosus pathogenesis (Review). Exp Ther Med 17: 1085-1090, 2019.

19. Raducan A, Bucur S, Caruntu C, Constantin T, Nita IE, Manolache $\mathrm{N}$ and Constantin MM: Therapeutic management with biological anti-TNF- $\alpha$ agent in severe psoriasis associated with chronic hepatitis B: A case report. Exp Ther Med 18: 895-899, 2019

20. Constantin MM, Cristea CM, Taranu T, Bucur S, Constantin T, Dinu A, Jinga $M$ and Nita IE: Biosimilars in dermatology: The wind of change. Exp Ther Med 18: 911-915, 2019

21. Raducan A, Bucur S, Caruntu C, Constantin T, Nita IE, Manolache N and Constantin MM: Therapeutic management with biological anti-TNF- $\alpha$ agent in severe psoriasis associated with chronic hepatitis B: A case report. Exp Ther Med 18: 895-899, 2019.

22. Olteanu R, Zota A and Constantin M: Biosimilars: An update on clinical trials (review of published and ongoing studies). Acta Dermatovenerol Croat 25: 57-66, 2017.

23. Gamret AC, Price A, Fertig RM, Lev-Tov H and Nichols AJ: Complementary and alternative medicine therapies for psoriasis: A systematic review. JAMA Dermatol 154: 1330-1337, 2018.

24. Nwabudike LC and Tatu AL: Using complementary and alternative medicine for the treatment of psoriasis: A step in the right direction. JAMA Dermatol 155: 636, 2019.

25. Nwabudike LC and Tatu A: Response to: Murphy EC, Nussbaum D, Prussick R, Friedman AJ: Use of complementary and alternative medicine by patients with psoriasis. J Am Acad Dermatol 81: e105, 2019.

26. Husted JA, Tom BD, Schentag CT, Farewell VT and Gladman DD: Occurrence and correlates of fatigue in psoriatic arthritis. Ann Rheum Dis 68: 1553-1558, 2009. 